\title{
PARAMETRIZED ARITY GAP
}

\author{
MIGUEL COUCEIRO, ERKKO LEHTONEN, AND TAMÁS WALDHAUSER
}

\begin{abstract}
We propose a parametrized version of arity gap. The parametrized arity gap $\operatorname{gap}(f, \ell)$ of a function $f: A^{n} \rightarrow B$ measures the minimum decrease in the number of essential variables of $f$ when $\ell$ consecutive identifications of pairs of essential variables are performed. We determine $\operatorname{gap}(f, \ell)$ for an arbitrary function $f$ and a nonnegative integer $\ell$. We also propose other variants of arity gap and discuss further problems pertaining to the effect of identification of variables on the number of essential variables of functions.
\end{abstract}

\section{INTRODUCTION}

Let $A$ and $B$ be arbitrary nonempty sets. In this paper we investigate a variant of the so-called arity gap of functions $f: A^{n} \rightarrow B$. The study of arity gap goes back to the 1963 paper by Salomaa [13, where he addressed the question how the number of essential variables of a function is affected by substitution of constants for variables or by identification of variables.

The arity gap of a function $f: A^{n} \rightarrow B$ is defined as the minimum decrease in the number of essential variables when any two essential variables of $f$ are identified, and it is denoted by gap $f$. Concerning the effect of identifying variables on the number of essential variables, Salomaa's main result asserts that in the case when $A=B=\{0,1\}$, it holds that gap $f \leq 2$ for every function $f:\{0,1\}^{n} \rightarrow\{0,1\}$ with at least two essential variables. (In fact, it is implicit in Salomaa's work that if $|A|=k$ and $|B| \geq 2$, then the arity gap of any function $f: A^{n} \rightarrow B$, all variables of which are essential, is at most $k$, and that there exist functions of arity $k$ for which this upper bound is met.)

For $|A|=2$, examples of functions meeting each of the two possible values of the arity gap can be easily constructed. For instance, it is clear that the Boolean multiplication has arity gap 1, whereas the Boolean addition has arity gap 2. This observation asks for a complete classification of Boolean functions into ones with arity gap 1 and ones with arity gap 2.

Such a classification was attained in 4, where explicit descriptions of functions with arity gap 2 were provided; interestingly, this result led to a similar classification of pseudo-Boolean functions (i.e., $A=\{0,1\}$ and $|B| \geq 2$ ) into ones with arity gap 1 and ones with arity gap 2 (see [5]).

Willard [16] extended Salomaa's result to functions defined on arbitrary finite domains and showed that the same upper bound 2 holds for the arity gap of any function $f: A^{n} \rightarrow B$ depending on all of its variables, provided that $n>\max (3,|A|)$. Moreover, he proved that the arity gap of such a function $f$ is 2 if and only if $f$ is determined by oddsupp (see Section 2.2).

Further classifications for wider classes of functions (e.g., where $A$ and $B$ are arbitrary nonempty sets) were obtained and made explicit under certain conditions in, e.g., [6, 7, 8, 14.

2010 Mathematics Subject Classification. 06A06, 08A40.

Key words and phrases. arity gap, parametrized arity gap, essential variable, simple minor, variable identification minor.

The first named author is supported by the internal research project F1R-MTH-PUL-09MRDO of the University of Luxembourg. The third named author acknowledges that the present project is supported by the TÁMOP-4.2.1/B-09/1/KONV-2010-0005 program of the National Development Agency of Hungary, by the Hungarian National Foundation for Scientific Research under grants no. K77409 and K83219, by the National Research Fund of Luxembourg, and cofunded under the Marie Curie Actions of the European Commission (FP7-COFUND).. 
In this paper we introduce a parametrized version of arity gap which measures the minimum decrease in the number of essential variables when we make $\ell \geq 1$ successive identifications of pairs of essential variables. For a function $f: A^{n} \rightarrow B$, let $\operatorname{gap}(f, \ell)$ denote this minimum decrease. This parametrized notion extends that of arity gap as $\operatorname{gap} f=\operatorname{gap}(f, 1)$.

This paper is organized as follows. In Section 2, we recall basic notions and establish preliminary results concerning the simple minor relation and the arity gap, which will be needed in the later sections. Section 3 is devoted to the study of the parametrized arity gap. In particular, given sets $A$ and $B$ and positive integers $n, p$, we explicitly determine the possible sequences

$$
\operatorname{gap}(f, 1), \operatorname{gap}(f, 2), \ldots, \operatorname{gap}(f, \ell), \ldots,
$$

for functions $f: A^{n} \rightarrow B$ depending on all of their variables such that gap $f=p$. In Section 4, we briefly discuss some further problems related to the effect of several identifications of essential variables on the number of essential variables of a function.

The current study was motivated by the questions and remarks made by Dan A. Simovici at the IEEE 41st International Symposium on Multiple-Valued Logic (ISMVL 2011).

\section{Preliminaries}

2.1. Functions of several variables and simple minors. For a positive integer $n$, we will denote $[n]:=\{1, \ldots, n\}$, and we will assume throughout this paper that $A$ and $B$ are arbitrary sets with at least two elements. A function of several variables from $A$ to $B$ is a map $f: A^{n} \rightarrow B$ for some positive integer $n$ called the arity of $f$. We denote the set of all finitary functions from $A$ to $B$ by

$$
\mathcal{F}_{A B}:=\bigcup_{n \geq 1} B^{A^{n}}
$$

We say that the $i$-th variable $x_{i}$ is essential in $f: A^{n} \rightarrow B$, or $f$ depends on $x_{i}$, if there exist tuples $\mathbf{a}:=\left(a_{1}, \ldots, a_{n}\right)$ and $\mathbf{b}:=\left(b_{1}, \ldots, b_{n}\right)$ such that $a_{j}=b_{j}$ for all $j \neq i$ and $f(\mathbf{a}) \neq f(\mathbf{b})$. A variable that is not essential is called inessential. The essential arity of $f$ is defined to be the cardinality of the set

$$
\text { Ess } f:=\left\{i \in[n]: x_{i} \text { is essential in } f\right\}
$$

and is denoted by ess $f$. If ess $f=n$, then we say that $f$ is essentially $n$-ary.

Let $f: A^{n} \rightarrow B$ and $g: A^{m} \rightarrow B$. We say that $g$ is a simple minor of $f$, and we write $g \leq f$, if there exists a map $\alpha:[n] \rightarrow[m]$ such that $g\left(a_{1}, \ldots, a_{m}\right)=f\left(a_{\alpha(1)}, \ldots, a_{\alpha(n)}\right)$ for all $a_{1}, \ldots, a_{m} \in A$. (Informally, $g$ is a simple minor of $f$, if $g$ can be obtained from $f$ by permutation of variables, addition of inessential variables, deletion of inessential variables, or identification of variables.)

Let $f: A^{n} \rightarrow B$. For $i, j \in[n], i \neq j$, the simple minor $f_{i \leftarrow j}: A^{n} \rightarrow B$ of $f$ given by the rule

$$
f_{i \leftarrow j}\left(a_{1}, \ldots, a_{n}\right)=f\left(a_{1}, \ldots, a_{i-1}, a_{j}, a_{i+1}, \ldots, a_{n}\right),
$$

for all $a_{1}, \ldots, a_{n} \in A$, is called an identification minor of $f$, obtained by substituting $x_{j}$ for $x_{i}$. Note that $a_{j}$ occurs twice on the right-hand side of the above equality while $a_{i}$ does not appear at all. Thus, $x_{i}$ is necessarily inessential in $f_{i \leftarrow j}$.

The simple minor relation $\leq$ is a quasiorder on $\mathcal{F}_{A B}$. As for quasiorders, $\leq$ induces an equivalence relation $\equiv$ on $\mathcal{F}_{A B}$. If $f \equiv g$, then we say that $f$ and $g$ are equivalent. (Informally, $f$ and $g$ are equivalent, if each of $f$ and $g$ can be obtained from the other by permutation of variables, addition of inessential variables, and deletion of inessential variables.)

Remark 2.1. If $f \equiv g$, then ess $f=\operatorname{ess} g$. Every nonconstant function is equivalent to a function that depends on all of its variables.

If $g \leq f$ but $f \not \equiv g$, then we write $g<f$ and say that $g$ is a strict minor of $f$. If $g<f$ but there is no $h$ such that $g<h<f$, then we say that $g$ is a lower cover of $f$ and denote this fact by $g \prec f$. 
Remark 2.2. It was shown in [2] that the lower covers of any function $f: A^{n} \rightarrow B$ have the same essential arity when $A=B=\{0,1\}$. The proof of this fact given in [2] actually shows that this claim is true whenever $|A|=2$ and $|B| \geq 2$. However, this is not the case when $|A|>2$, as the following example illustrates.

Example 2.3. Let $A$ be a set with at least three elements, let $B$ be a set with at least two elements, and assume that 0 and 1 are distinct elements of $B$. Let $\nu: A^{2} \rightarrow B$ be the inequality predicate

$$
\nu(x, y)= \begin{cases}1, & \text { if } x \neq y \\ 0, & \text { if } x=y\end{cases}
$$

and let $\wedge: B^{2} \rightarrow B$ and $\vee: B^{2} \rightarrow B$ be arbitrary extensions of the Boolean conjunction and disjunction to $B$ (i.e., arbitrary binary operations on $B$ satisfying $0 \wedge 0=0 \wedge 1=$ $1 \wedge 0=0,1 \wedge 1=1,0 \vee 0=0,1 \vee 0=0 \vee 1=1 \vee 1=1)$. Consider the function $f: A^{4} \rightarrow B$ defined by

$$
f\left(x_{1}, x_{2}, x_{3}, x_{4}\right):=\nu\left(x_{1}, x_{2}\right) \vee \bigwedge_{\substack{1 \leq i<j \leq 4 \\(i, j) \neq(1,2)}} \nu\left(x_{i}, x_{j}\right) .
$$

It is easy to see that $f_{i \leftarrow j}=\nu\left(x_{1}, x_{2}\right)$ for $1 \leq i<j \leq 4,(i, j) \neq(1,2)$, and

$$
f_{1 \leftarrow 2}=\bigwedge_{2 \leq i<j \leq 4} \nu\left(x_{i}, x_{j}\right) .
$$

Furthermore, ess $f=4$, ess $f_{1 \leftarrow 2}=3$, ess $f_{i \leftarrow j}=2$, and $f_{1 \leftarrow 2} \not \leq f_{i \leftarrow j} \not \leq f_{1 \leftarrow 2}$, for every $1 \leq i<j \leq 4,(i, j) \neq(1,2)$. Hence, $f$ has two lower covers of different essential arities.

For background on the simple minor relation and its variants, see [3, 19, 10, 11, 12 15. 17.

We say that $f$ is totally symmetric, if for all permutations $\pi$ of $[n]$ the identity $f\left(a_{1}, \ldots, a_{n}\right)=f\left(a_{\pi(1)}, \ldots, a_{\pi(n)}\right)$ holds for all $a_{1}, \ldots, a_{n} \in A$. Observe that a totally symmetric function depends on either all or none of its variables.

Fact 2.4. If $f: A^{n} \rightarrow B$ is totally symmetric, then for all $i, j, i^{\prime}, j^{\prime} \in[n]\left(i \neq j, i^{\prime} \neq j^{\prime}\right)$, $f_{i \leftarrow j} \equiv f_{i^{\prime} \leftarrow j^{\prime}}$. Therefore, if $f$ is nonconstant, then for all distinct $i, j \in[n], f_{i \leftarrow j}$ is, up to equivalence, the unique lower cover of $f$.

2.2. Functions determined by supp and oddsupp. Following Berman and Kisielewicz [1], we define supp: $\bigcup_{n \geq 1} A^{n} \rightarrow \mathcal{P}(A)$ and oddsupp: $\bigcup_{n \geq 1} A^{n} \rightarrow \mathcal{P}(A)$ as

$$
\begin{aligned}
\operatorname{supp}\left(a_{1}, \ldots, a_{n}\right) & :=\left\{a_{1}, \ldots, a_{n}\right\}, \\
\operatorname{odd} \operatorname{supp}\left(a_{1}, \ldots, a_{n}\right) & :=\left\{a \in A:\left|\left\{i \in[n]: a_{i}=a\right\}\right| \text { is odd }\right\} .
\end{aligned}
$$

We say that $f: A^{n} \rightarrow B$ is determined by supp (respectively, determined by oddsupp), if there exists a function $\varphi: \mathcal{P}(A) \rightarrow B$ such that $f=\left.\varphi \circ \operatorname{supp}\right|_{A^{n}}$ (respectively, $f=\varphi$ o oddsupp $\left.\mid A^{n}\right)$. Note that every function determined by supp or oddsupp is totally symmetric; hence such a function either depends on all of its variables or on none of them. However, not every totally symmetric function is determined by supp or oddsupp.

Remark 2.5. For any positive integer $n$, let us define the following subsets of $\mathcal{P}(A)$ :

$$
\begin{aligned}
& \mathcal{P}_{\leq n}(A):=\{S \subseteq A: 1 \leq|S| \leq n\}, \\
& \mathcal{P}_{\leq n}^{\prime}(A):=\{S \subseteq A:|S| \in\{n, n-2, \ldots\}\} .
\end{aligned}
$$

Clearly, $\left\{\operatorname{supp}(\mathbf{x}): \mathbf{x} \in A^{n}\right\}=\mathcal{P}_{\leq n}(A)$, therefore the restriction of $\varphi: \mathcal{P}(A) \rightarrow B$ to $\mathcal{P}_{\leq n}(A)$ uniquely determines the function $\varphi \circ \operatorname{supp} \mid A^{n}$, and vice versa. Similarly, we have $\left\{\operatorname{oddsupp}(\mathbf{x}): \mathbf{x} \in A^{n}\right\}=\mathcal{P}_{\leq n}^{\prime}(A)$, and consequently there is a one-to-one correspondence between functions $f: A^{n} \rightarrow B$ determined by oddsupp and maps $\varphi: \mathcal{P}_{\leq n}^{\prime}(A) \rightarrow B$. In particular, $\left.\varphi \circ \operatorname{supp}\right|_{A^{n}}\left(\operatorname{resp} . \varphi \circ\right.$ oddsupp $\left.\left.\right|_{A^{n}}\right)$ is constant if and only if $\left.\varphi\right|_{\mathcal{P}_{\leq n}(A)}$ (resp. $\left.\varphi\right|_{\mathcal{P}_{\leq n}^{\prime}(A)}$ ) is constant, and has essential arity $n$ otherwise. 
Example 2.6. Every constant function and every unary function is determined by both supp and oddsupp. Furthermore, for each $2 \leq n \leq|A|$, there are nonconstant functions $f: A^{n} \rightarrow B$ that are determined by both supp and oddsupp. For instance, let $a$ and $b$ be distinct elements of $B$ and define $f: A^{n} \rightarrow B$ by the rule

$$
f\left(a_{1}, \ldots, a_{n}\right):= \begin{cases}a & \text { if } a_{i} \neq a_{j} \text { for all } i \neq j, \\ b & \text { otherwise. }\end{cases}
$$

It is easy to see that $f=\left.\phi \circ \operatorname{supp}\right|_{A^{n}}=\phi \circ$ oddsupp $\left.\right|_{A^{n}}$, where $\phi: \mathcal{P}(A) \rightarrow B$ is the map

$$
\phi(S):= \begin{cases}a & \text { if }|S|=n \\ b & \text { otherwise. }\end{cases}
$$

Our next result shows that, in fact, such nontrivial examples of functions determined by both supp and oddsupp can only be found among functions with small arities.

Proposition 2.7. If $n>|A|$, then $f: A^{n} \rightarrow B$ is determined by both supp and oddsupp if and only if $f$ is a constant function.

Proof. The condition is clearly sufficient: as noted in Example 2.6. every constant function is determined by both supp and oddsupp.

For necessity, assume that $f$ is determined by both supp and oddsupp. Then there exist maps $\varphi, \psi: \mathcal{P}(A) \rightarrow B$ such that $f=\varphi \circ \operatorname{supp} \mid A^{n}=\psi \circ$ oddsupp $\left.\right|_{A^{n}}$. We will prove that for every $S \subseteq A(|S| \geq 2), t \in S$, it holds that $\varphi(S)=\varphi(S \backslash\{t\})$. A simple inductive argument then shows that the restriction of $\varphi$ to $\mathcal{P}(A) \backslash\{\emptyset\}$ is constant, which implies that $f$ is constant, as claimed.

Thus, let $S \subseteq A,|S|=s \geq 2$, say $S=\left\{a_{1}, \ldots, a_{s}\right\}, a_{s}=t$. Since $|S| \leq|A|<n$, there exists an injective map $h: S \rightarrow[n]$. We will define tuples $\mathbf{b}, \mathbf{c} \in A^{n}$; the definition depends on the parity of $n-|S|$ :

- If $n-|S|$ is odd, then for each $j \in[n]$, let

$$
b_{j}:=\left\{\begin{array}{ll}
a_{i} & \text { if } h\left(a_{i}\right)=j, \\
a_{s} & \text { otherwise, }
\end{array} \quad c_{j}:= \begin{cases}a_{i} & \text { if } h\left(a_{i}\right)=j, i \neq s, \\
a_{s-1} & \text { otherwise. }\end{cases}\right.
$$

Since $n-|S|$ is odd, $\mathbf{b}$ has an even number of occurrences of $a_{s}$, and $\mathbf{c}$ has an odd number of occurrences of $a_{s-1}$. Therefore $\operatorname{supp}(\mathbf{b})=S, \operatorname{supp}(\mathbf{c})=\operatorname{oddsupp}(\mathbf{b})=$ $\operatorname{oddsupp}(\mathbf{c})=S \backslash\left\{a_{s}\right\}$

- If $n-|S|$ is even, then for each $j \in[n]$, let

$$
b_{j}:=\left\{\begin{array}{ll}
a_{i} & \text { if } h\left(a_{i}\right)=j, i \neq s, \\
a_{s-1} & \text { if } h\left(a_{s}\right)=j, \\
a_{s} & \text { otherwise, }
\end{array} \quad c_{j}:= \begin{cases}a_{i} & \text { if } h\left(a_{i}\right)=j, i \neq s, \\
a_{s-1} & \text { otherwise. }\end{cases}\right.
$$

Since $n-|S|$ is even, $\mathbf{b}$ has two occurrences of $a_{s-1}$ and an even number of occurrences of $a_{s}$, and $\mathbf{c}$ has an even number of occurrences of $a_{s-1}$. Therefore $\operatorname{supp}(\mathbf{b})=S$, $\operatorname{supp}(\mathbf{c})=S \backslash\left\{a_{s}\right\}, \operatorname{oddsupp}(\mathbf{b})=\operatorname{oddsupp}(\mathbf{c})=S \backslash\left\{a_{s-1}, a_{s}\right\}$.

Our choice of $\mathbf{b}$ and $\mathbf{c}$ yields

$$
\varphi \circ \operatorname{supp}(\mathbf{b})=f(\mathbf{b})=\psi \circ \operatorname{oddsupp}(\mathbf{b})=\psi \circ \operatorname{oddsupp}(\mathbf{c})=f(\mathbf{c})=\varphi \circ \operatorname{supp}(\mathbf{c}),
$$

that is, $\varphi(S)=\varphi\left(S \backslash\left\{a_{s}\right\}\right)$. This completes the proof of the proposition.

As it will become clear from Propositions 2.8 and 2.11 below, if a nonconstant function $f$ is determined by oddsupp (supp, respectively) then every simple minor of $f$ is equivalent to a function that is determined by oddsupp (supp, respectively).

Proposition 2.8. Let $A$ and $B$ be finite nonempty sets, and let $k:=|A|$. If $f: A^{n} \rightarrow B$ is a nonconstant function determined by oddsupp, then the simple minors of $f$ form a chain

$$
f=f_{n} \succ f_{n-2} \succ \cdots \succ f_{n-2 t-2} \succ f_{n-2 t}
$$

of length $t$ such that ess $f_{n-2 i}=n-2 i$ for all $i<t$. Moreover, we either have ess $f_{n-2 t}=$ 1 and $t=\frac{n-1}{2}$ or ess $f_{n-2 t}=0$ and $\left\lceil\frac{n-k}{2}\right\rceil<t \leq\left\lfloor\frac{n}{2}\right\rfloor$. 
Proof. Let us assume that $f=\varphi$ o oddsupp $\left.\right|_{A^{n}}$ for some $\varphi: \mathcal{P}(A) \rightarrow B$. Since $f$ is totally symmetric, it has a unique lower cover, namely the $(n-2)$-ary function $f_{n-2}$ given by $f_{n-2}=\varphi$ o oddsupp $\left.\right|_{A^{n-2}}$. We see that $f_{n-2}$ is also determined by oddsupp, hence we can repeat this argument, and we conclude that the simple minors of $f$ form a chain as stated in the theorem. It is obvious that the length of this chain is $t \leq\left\lfloor\frac{n}{2}\right\rfloor$. As every function in this chain is totally symmetric, the essential arity of $f_{n-2 i}$ is either $n-2 i$ or 0 for all $i \leq t$. Clearly, only the last element of the chain can be constant, i.e., ess $f_{n-2 i}=n-2 i$ for $i<t$. Moreover, $f_{n-2 t}$ has no proper simple minor, therefore it is essentially at most unary. If ess $f_{n-2 t}=1$, then $n-2 t=1$, thus $t=\frac{n-1}{2}$, as claimed.

Now let us assume that ess $f_{n-2 t}=0$. By Remark 2.5, $f_{n-2 i}$ is not constant if and only if the restriction of $\varphi$ to $\mathcal{P}_{\leq n-2 i}^{\prime}(A)$ is not constant. Since $f$ is not constant, and $\mathcal{P}_{\leq n}^{\prime}(A)=\mathcal{P}_{\leq n-2 i}^{\prime}(A)$ whenever $n-2 i+2>k$, we have that $f_{n-2 i}$ is not constant for all $i \leq\left\lceil\frac{n-k}{2}\right\rceil$. As $f_{n-2 t}$ is constant by our assumption, it follows that $t>\left\lceil\frac{n-k}{2}\right\rceil$.

In the following two examples we construct for all possible values of $k, n$ and $t$ a function determined by oddsupp whose simple minors form a chain as stated in Proposition 2.8, thereby showing that this result cannot be sharpened.

Example 2.9. Let $k, n, t$ be positive integers such that $k \geq 2$ and $\left\lceil\frac{n-k}{2}\right\rceil<t \leq\left\lfloor\frac{n}{2}\right\rfloor$. Then $s:=n-2 t+2$ satisfies the inequalities $2 \leq s \leq k$. Let $A$ be a set with $k$ elements, let $B$ be a set with at least two elements, and let us define $\varphi: \mathcal{P}(A) \rightarrow B$ by

$$
\varphi(S):= \begin{cases}1 & \text { if }|S| \geq s \\ 0 & \text { if }|S|<s\end{cases}
$$

where 0 and 1 denote two distinct elements of $B$. For every $0 \leq i \leq t$, let $f_{n-2 i}: A^{n-2 i} \rightarrow$ $B$ be the function determined by oddsupp via $\varphi$, i.e., let $f_{n-2 i}=\varphi$ o oddsupp $\left.\right|_{A^{n-2 i}}$. It is straightforward to verify with the help of Remark 2.5 that $f_{n-2 t}=f_{s-2}$ is constant, and that ess $f_{n-2 i}=n-2 i$ if $i<t$. Moreover, for $i<t$, the unique lower cover of $f_{n-2 i}$ is $f_{n-2 i-2}$. Thus the simple minors of $f_{n}$ form a chain exactly as in (the second case of) Proposition 2.8

Example 2.10. Let $k, n, t$ be positive integers such that $k \geq 2$ and $t=\frac{n-1}{2}$. Let $A$ be a set with $k$ elements, let $B$ be a set with at least two elements, and let $\varphi: \mathcal{P}(A) \rightarrow B$ be any function that is not constant on singletons, i.e., there exist $a_{1}, a_{2} \in A$ such that $\varphi\left(\left\{a_{1}\right\}\right) \neq \varphi\left(\left\{a_{2}\right\}\right)$. For every odd number $r$, let $f_{r}: A^{r} \rightarrow B$ be the function determined by oddsupp via $\varphi$, i.e., let $f_{r}=\varphi$ o oddsupp $\left.\right|_{A^{r}}$. Then $f_{r}$ is not constant, hence ess $f_{r}=r$, and the unique lower cover of $f_{r}$ is $f_{r-2}$. Thus the simple minors of $f_{n}$ form a chain exactly as in (the first case of) Proposition 2.8

Reasoning as above, by making use of Remark 2.5, we have the following analogue of Proposition 2.8

Proposition 2.11. Let $A$ and $B$ be finite nonempty sets, and let $k:=|A|$. If $f: A^{n} \rightarrow$ $B$ is a nonconstant function determined by supp, then the simple minors of $f$ form a chain

$$
f=f_{n} \succ f_{n-1} \succ \cdots \succ f_{n-t+1} \succ f_{n-t}
$$

of length $t$ such that ess $f_{n-i}=n-i$ for all $i<t$. Moreover, we either have ess $f_{n-t}=1$ and $t=n-1$, or ess $f_{n-t}=0$ and $n-k<t<n$.

As for functions determined by oddsupp, we can find functions which fall into each of the two possible cases provided in Proposition 2.11.

2.3. Arity gap. Let $f: A^{n} \rightarrow B$ be a function that depends on at least two variables, i.e., ess $f \geq 2$. The arity gap of $f$, denoted gap $f$, is defined as

$$
\text { gap } f:=\min _{\substack{i, j \in \operatorname{Ess} f \\ i \neq j}}\left(\operatorname{ess} f-\operatorname{ess} f_{i \leftarrow j}\right) \text {. }
$$


While $f_{i \leftarrow j}$ is not necessarily a lower cover of $f$ in the simple minor quasiorder, every lower cover of $f$ is of the form $f_{i \leftarrow j}$. Therefore, we could define the arity gap of $f$ in an equivalent way as

$$
\text { gap } f:=\min _{g \prec f}(\operatorname{ess} f-\operatorname{ess} g) \text {. }
$$

Whenever we consider the arity gap of a function $f: A^{n} \rightarrow B$, we may assume, without loss of generality, that $f$ depends on all of its variables (see Remark 2.1).

As made apparent by Willard [16], the notion of arity gap is tightly related to determinability by supp and oddsupp. The following corollaries are immediate consequences of Propositions 2.8 and 2.11.

Corollary 2.12. If $f: A^{n} \rightarrow B$ is determined by oddsupp, $n>|A|$, and $f$ is nonconstant, then gap $f=2$.

Corollary 2.13. If $f: A^{n} \rightarrow B$ is determined by supp, $n>|A|$, and $f$ is nonconstant, then gap $f=1$.

We now recall a few noteworthy results about the arity gap. These appear as Lemma 1.2, Lemma 2.2, Corollary 2.3, and Corollary 2.7 in Willard's paper [16].

Theorem 2.14 (Willard [16]). Let $A$ and $B$ be finite nonempty sets, and let $k:=|A|$. Suppose that $f: A^{n} \rightarrow B$ depends on all of its variables. If $n>k$, then gap $f \leq 2$. Moreover, if $n>\max (k, 3)$, then gap $f=2$ if and only if $f$ is determined by oddsupp.

Lemma 2.15 (Willard [16]). Let $A$ and $B$ be finite nonempty sets, and let $k:=|A|$. Suppose that $f: A^{n} \rightarrow B$ depends on all of its variables.

(1) If $n>2$, gap $f=1, f$ is totally symmetric, and for any distinct $i, j \in[n], f_{i \leftarrow j}$ is equivalent to a totally symmetric function, then $f$ is determined by supp.

(2) If $f$ is determined by supp, then $f_{i \leftarrow j}$ is equivalent to a function determined by supp for any distinct $i, j \in[n]$. Moreover, if $n>k$, then $f_{i \leftarrow j}$ is nonconstant.

(3) If $n \geq \max (k, 3)+2$ and $f$ is not totally symmetric, then there exist distinct $i, j \in[n]$ such that $f_{i \leftarrow j}$ depends on $n-1$ variables and is not equivalent to a totally symmetric function.

The following theorem will play an important role in the next section.

Theorem 2.16. Let $A$ and $B$ be finite nonempty sets, and let $k:=|A|$. Suppose that $f: A^{n} \rightarrow B$ depends on all of its variables.

(1) If $n \geq \max (k, 3)+1$ and gap $f=2$, then for all $g<f$ with ess $g>k$, it holds that gap $g=2$.

(2) If $n \geq \max (k, 3)+2$ and gap $f=1$, then there exists a $g \prec f$ such that gap $g=1$ and ess $g=n-1$.

Proof. For (1), observe that Theorem 2.14 and the assumption gap $f=2$ imply that $f$ is determined by oddsupp. Hence, by Proposition 2.8, $g$ is also determined by oddsupp. By Corollary 2.12, gap $g=2$.

For (2), observe that Theorem 2.14 and the assumption gap $f=1$ imply that $f$ is not determined by oddsupp. If $f$ is not totally symmetric, then the claim holds by Lemma 2.15 (3) and Theorem 2.14. Therefore we may assume that $f$ is totally symmetric. By Fact 2.4, $f$ has a unique lower cover $g$, and ess $g=\operatorname{ess} f-1$. If $g$ is not totally symmetric, then it is not determined by oddsupp, and by Theorem 2.14. gap $g=1$. If $g$ is totally symmetric, then Lemma 2.15 (10) implies that $f$ is determined by supp. By Lemma 2.15 (2), $g$ is determined by supp. By Proposition 2.7 $g$ is not determined by oddsupp, from which it follows that gap $g=1$ by Theorem 2.14 .

\section{Parametrized ARIty GaP}

In this section we are interested in the following parametrized version of arity gap which measures the minimum decrease in the essential arity when we take $\ell \geq 0$ steps downwards in the simple minor partial order:

$$
\operatorname{gap}(f, \ell):=\min _{g \in \downarrow^{\ell} f}(\operatorname{ess} f-\operatorname{ess} g),
$$


where $\downarrow^{0} f:=\left\{g \in \mathcal{F}_{A B}: g \equiv f\right\}$ and, for $\ell>0$,

$$
\downarrow^{\ell} f:=\left\{g \in \mathcal{F}_{A B} \mid \exists f_{1}, \ldots, f_{\ell-1}: f \succ f_{1} \succ \cdots \succ f_{\ell-1} \succ g\right\} .
$$

Note that $\operatorname{gap}(f, \ell)$ is defined only if there exists a chain of length $\ell$ below $f$, and in this case $\ell \leq \operatorname{gap}(f, \ell) \leq \operatorname{ess} f$. The arity gap (as defined in Section 2.3) corresponds to the case $\ell=1$ : we have $\operatorname{gap}(f, 1)=\operatorname{gap} f$ for every function $f$. Observe also that $\operatorname{gap}(f, 0)=0$ for every function $f$.

We saw in Section 2.1 that taking a strict minor of a function $f$ requires the identification of at least one pair of essential variables of $f$; otherwise, the minors we obtain are equivalent to $f$. This means that $\operatorname{gap}(f, \ell)$ can be computed by sequentially identifying a pair of essential variables $\ell$ times in all possible ways, starting from $f$, and then determining the sequence of $\ell$ identifications which results in the minimum loss of essential variables.

Remark 3.1. It is worth stressing the fact that the identification of variables is performed sequentially, and at each step only one pair of essential variables is identified; otherwise, ambiguities could occur since a priori we do not know which essential variables become inessential after a pair is identified.

We mentioned in Section 2.3 that not every identification minor $f_{i \leftarrow j}$ is a lower cover of $f$, and if $f_{i \leftarrow j}$ is not a lower cover of $f$, then gap $f<\operatorname{ess} f-\operatorname{ess} f_{i \leftarrow j}$. Moreover, it can be the case that $f$ has two lower covers $f_{1}$ and $f_{2}$ such that ess $f_{1}<\operatorname{ess} f_{2}$, and again we would conclude that gap $f<\operatorname{ess} f-\operatorname{ess} f_{1}$. Hence, one might be led to thinking that in order to compute $\operatorname{gap}(f, \ell)$ it suffices to choose at each recursion step an identification which results in the minimum loss of essential variables. However, as the following example illustrates, this is not true.

Example 3.2. Let $A$ be the 5-element field and consider the polynomial function $f: A^{6} \rightarrow A$ defined by:

$$
f\left(x_{1}, x_{2}, x_{3}, x_{4}, x_{5}, x_{6}\right):=\left(x_{1}-x_{2}\right)\left(x_{5}-x_{6}\right)+\prod_{\substack{1 \leq i<j \leq 6 \\(i, j) \neq(5,6)}}\left(x_{i}-x_{j}\right) .
$$

It is easy to verify that ess $f=6, f_{1 \leftarrow 2} \equiv 0$, and that $f$ has, up to equivalence, two lower covers, namely,

$$
\begin{aligned}
& f_{3 \leftarrow 1}=\left(x_{1}-x_{2}\right) \cdot\left(x_{5}-x_{6}\right), \\
& f_{6 \leftarrow 5}=\prod_{1 \leq i<j \leq 4}\left(x_{i}-x_{j}\right) \cdot \prod_{1 \leq i \leq 4}\left(x_{i}-x_{5}\right)^{2} .
\end{aligned}
$$

Figure 1 presents the Hasse diagram of the principal ideal generated by the equivalence class of $f$ in the simple minor poset. The label of each edge $g \prec h$ is the number ess $h$ - ess $g$. We use the following notation for the simple minors of $f$ :

$$
\begin{array}{ll}
q_{1}=\prod_{1 \leq i<j \leq 4}\left(x_{i}-x_{j}\right) \cdot \prod_{1 \leq i \leq 4}\left(x_{i}-x_{5}\right)^{2}, & q_{2}=\left(x_{1}-x_{2}\right) \cdot\left(x_{3}-x_{4}\right), \\
q_{3}=\left(x_{1}-x_{2}\right) \cdot\left(x_{1}-x_{3}\right), & q_{4}=\left(x_{1}-x_{2}\right) \cdot\left(x_{2}-x_{3}\right), \\
q_{5}=\left(x_{1}-x_{2}\right)^{2}, & q_{6}=-\left(x_{1}-x_{2}\right)^{2} .
\end{array}
$$

Now if we would choose as our first identification the pair $\{5,6\}$, then any other identification of essential variables results in the loss of all the remaining essential variables. In other words, any downward path in Figure 1 which starts from $f$ and passes through $q_{1}$ has length 2 , and along it we first lose 1 and then 5 essential variables. However, the downward paths that start from $f$ and pass through $q_{2}$ have length 4 , and along them we lose $2,1,1$, and then 2 essential variables. This shows that, in order to compute $\operatorname{gap}(f, 1)$ as in (3.1), the minimum value is attained at the lower cover $q_{1}$, whereas, for $2 \leq \ell \leq 4$, we need to pass through $q_{2}$ for computing $\operatorname{gap}(f, \ell)$. Hence, $\operatorname{gap}(f, 0)=0$, $\operatorname{gap}(f, 1)=1, \operatorname{gap}(f, 2)=3, \operatorname{gap}(f, 3)=4$, and $\operatorname{gap}(f, 4)=6$. 


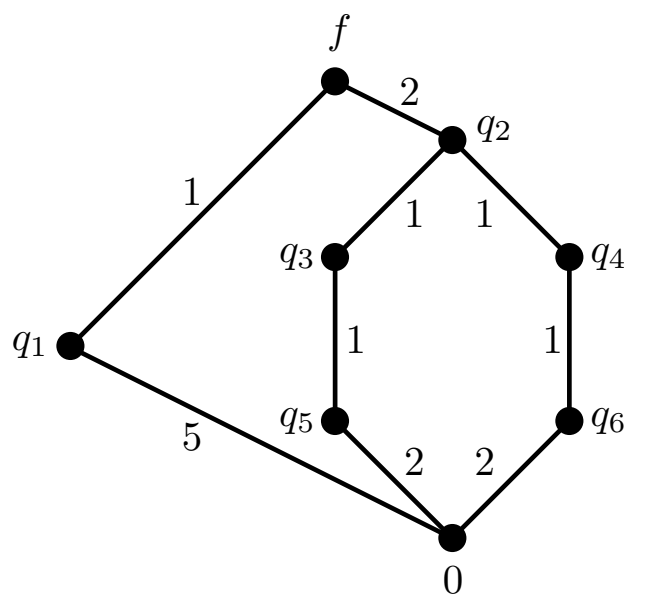

FigurE 1. The simple minors of the function $f$ given in Example 3.2

The following recursion formula is an immediate consequence of the definition:

$$
\operatorname{gap}(f, \ell)=\min _{g \prec f}(\operatorname{ess} f-\operatorname{ess} g+\operatorname{gap}(g, \ell-1)) .
$$

Theorem 3.3. Let $A$ and $B$ be finite nonempty sets, and let $k:=|A|$. Let $f: A^{n} \rightarrow B$, ess $f=n$ and $\operatorname{gap} f=1$. If $1 \leq \ell \leq n-\max (k, 3)$, then $\operatorname{gap}(f, \ell)=\ell$.

Proof. We prove the theorem by induction on $\ell$. If $\ell=1$, then $\operatorname{gap}(f, \ell)=\operatorname{gap} f=1$. If $\ell \geq 2$ then $n \geq \max (k, 3)+2$, hence by Theorem 2.16] $f$ has a lower cover $g$ with ess $g=n-1$ and gap $g=1$. Since $1 \leq \ell-1 \leq \operatorname{ess} g-\max (k, 3)$, we can apply the induction hypothesis to obtain $\operatorname{gap}(g, \ell-1)=\ell-1$. From (3.2) it follows that

$$
\operatorname{gap}(f, \ell) \leq \operatorname{ess} f-\operatorname{ess} g+\operatorname{gap}(g, \ell-1)=n-(n-1)+(\ell-1)=\ell .
$$

On the other hand we have the trivial inequality $\operatorname{gap}(f, \ell) \geq \ell$, and therefore $\operatorname{gap}(f, \ell)=$ $\ell$.

Informally, the above theorem means that if gap $f=1$, then we can walk down from $f$ in the simple minor quasiorder in such a way that in each step we lose only one essential variable, provided that the walk is not too long. The next result asserts that if we consider arbitrarily long walks, then we can lose any number of essential variables. More precisely, for each $2 \leq \ell \leq q \leq n$ we can find a function $f$ with ess $f=n$, gap $f=1$ and $\operatorname{gap}(f, \ell)=q$.

Theorem 3.4. For every $2 \leq \ell \leq q \leq n$, there exist sets $A$ and $B$ and a function $f: A^{n} \rightarrow B$ such that ess $f=n$, gap $f=1, \operatorname{gap}(f, \ell)=q,|A|<n$.

Proof. Let $p:=n-q, k:=n+1-\ell, A:=[k], B:=[3]$, and for $k \leq m \leq n$, define $f_{m}: A^{m} \rightarrow B$ as

$$
f_{m}\left(x_{1}, \ldots, x_{m}\right)= \begin{cases}1, & \text { if } x_{k}=x_{k+1}=\cdots=x_{m} \text { and } \operatorname{supp}(\mathbf{x})=A \\ 2, & \text { if } x_{1}=\cdots=x_{p}=2 \text { and } \operatorname{supp}(\mathbf{x}) \subset A \\ 3, & \text { otherwise. }\end{cases}
$$

We are going to prove that $\operatorname{gap}\left(f_{n}, \ell\right)=q$.

Claim 3.4.1. For $k \leq m \leq n$, the function $f_{m}$ depends on all of its variables.

Proof of Claim 3.4.1 Let $\mathbf{x}=(1,2, \ldots, k, k, \ldots, k) \in A^{m}$. Then $f_{m}(\mathbf{x})=1$, and it is easy to verify that if any coordinate of $\mathbf{x}$ is changed, the value of $f$ will change.

Claim 3.4.2. If $m>k$, then $f_{m-1} \prec f_{m}$.

Proof of Claim 3.4.2 Clearly $f_{m-1}\left(x_{1}, \ldots, x_{m-1}\right)=f_{m}\left(x_{1}, \ldots, x_{m-1}, x_{m-1}\right)$, so $f_{m-1} \leq$ $f_{m}$. Since ess $f_{m-1}=m-1$ and ess $f_{m}=m$ by Claim 3.4.1, $f_{m-1}$ is necessarily a lower cover of $f_{m}$. 
Claim 3.4.3. $f_{k}$ has a unique lower cover $u: A^{p} \rightarrow B$, and ess $u=p$.

Proof of Claim 3.4.3 Let $g=\left(f_{k}\right)_{i \leftarrow j}$; we may assume without loss of generality that $j<i$. Then

$$
g\left(x_{1}, \ldots, x_{k}\right)=f_{k}\left(x_{1}, \ldots, x_{i-1}, x_{j}, x_{i+1}, \ldots, x_{k}\right) .
$$

Since $\operatorname{supp}\left(x_{1}, \ldots, x_{i-1}, x_{j}, x_{i+1}, \ldots, x_{k}\right) \subset A$, we have that, if $i>p$,

$$
g\left(x_{1}, \ldots, x_{k}\right)= \begin{cases}2, & \text { if } x_{1}=\cdots=x_{p}=2 \\ 3, & \text { otherwise }\end{cases}
$$

and if $i \leq p$,

$$
g\left(x_{1}, \ldots, x_{k}\right)= \begin{cases}2, & \text { if } x_{1}=\cdots=x_{i-1}=x_{i+1}=\cdots=x_{p}=2, \\ 3, & \text { otherwise. }\end{cases}
$$

In the former case, $g$ is equivalent to the function $u: A^{p} \rightarrow B$,

$$
u\left(x_{1}, \ldots, x_{p}\right)= \begin{cases}2, & \text { if } x_{1}=\cdots=x_{p}=2, \\ 3, & \text { otherwise }\end{cases}
$$

and in the latter case $g$ is equivalent to the function $u^{\prime}: A^{p-1} \rightarrow B$,

$$
u^{\prime}\left(x_{1}, \ldots, x_{p-1}\right)= \begin{cases}2, & \text { if } x_{1}=\cdots=x_{p-1}=2, \\ 3, & \text { otherwise }\end{cases}
$$

It clearly holds that $u^{\prime}<u$, and we conclude that $u$ is the unique lower cover of $f_{k}$. $\diamond$

Claim 3.4.4. If $v<f$ and ess $v=t \leq k-1$, then $v \leq u$.

Proof of Claim 3.4.4 There exists $\sigma:[n] \rightarrow[t]$ such that

$$
v\left(x_{1}, \ldots, x_{t}\right)=f\left(x_{\sigma(1)}, \ldots, x_{\sigma(n)}\right) .
$$

Since $\operatorname{supp}\left(x_{\sigma(1)}, \ldots, x_{\sigma(n)}\right) \subset A$, we have

$$
f\left(x_{\sigma(1)}, \ldots, x_{\sigma(n)}\right)=u\left(x_{\sigma(1)}, \ldots, x_{\sigma(p)}\right) .
$$

Hence $v \leq u$.

By Claims 3.4.2 and 3.4.3, we have $f_{n} \succ f_{n-1} \succ \cdots \succ f_{k+1} \succ f_{k} \succ u$. Since $n-k+1=\ell$ and ess $f_{n}-\operatorname{ess} u=n-p=q$, it holds that $\operatorname{gap}\left(f_{n}, \ell\right) \leq q$. Suppose, on the contrary, that $\operatorname{gap}\left(f_{n}, \ell\right)<q$. Then there exists a chain $f_{n} \succ g_{1} \succ g_{2} \succ \cdots \succ g_{\ell}$ such that ess $f_{n}-\operatorname{ess} g_{\ell}<q$. Clearly ess $f_{n}-\operatorname{ess} g_{\ell} \geq \ell$, that is ess $g_{\ell} \leq \operatorname{ess} f_{n}-\ell=n-\ell=$ $k-1$. By Claim 3.4.4 $g_{\ell} \leq u$, whence ess $g_{\ell} \leq \operatorname{ess} u=p=n-q=\operatorname{ess} f_{n}-q<\operatorname{ess} g_{\ell}$. We have reached a contradiction that completes the proof of the theorem.

Next we consider the analogue of Theorem 3.3 for the case gap $f=2$.

Theorem 3.5. Let $A$ and $B$ be finite nonempty sets, and let $k:=|A|$. Let $f: A^{n} \rightarrow B$, ess $f=n$ and gap $f=2$. If $1 \leq \ell \leq\left\lceil\frac{n-k}{2}\right\rceil$, then $\operatorname{gap}(f, \ell)=2 \ell$.

Proof. If $\ell=1$, then $\operatorname{gap}(f, \ell)=\operatorname{gap} f=2=2 \ell$. If $\ell \geq 2$ then $n \geq k+3 \geq$ $\max (k, 3)+2$. Hence by Theorem $2.14 f$ is determined by oddsupp. The theorem then follows immediately from Proposition 2.8 .

Our last result shows that for almost every integer sequence $0=n_{0}<n_{1}<n_{2}<$ $\cdots<n_{r} \leq n$, we can construct a function $f: A^{n} \rightarrow B$ whose parametrized arity gap meets every member of the sequence. We only need to assume that $n_{r-1} \neq n-1$, because no function has both an essentially unary function and a constant function as its simple minors.

Theorem 3.6. Let $A$ be a finite set with $k$ elements and let $B$ be a set with at least two elements. Let $2 \leq n \leq k, 1 \leq r \leq n-1,0=n_{0}<n_{1}<n_{2}<\cdots<n_{r} \leq n$ such that $n-1 \leq n_{r} \leq n$ and $n_{r-1} \neq n-1$. Then there exists a function $f: A^{n} \rightarrow B$ such that $\operatorname{gap}(f, \ell)=n_{\ell}$ for every $0 \leq \ell \leq r$. 
Proof. We can assume that the codomain $B$ has a group structure, and 0 is the neutral element of the group operation + on $B$. We will define recursively functions $f_{i}: A^{n-n_{i}} \rightarrow B, 0 \leq i \leq r$, such that $f_{0} \succ f_{1} \succ \cdots \succ f_{r}$ and ess $f_{i}=n-n_{i}$ for all $0 \leq i \leq r$.

If $n_{r}=n-1$, then let $f_{r}: A \rightarrow B$ be an arbitrary nonconstant function. If $n_{r}=n$, then let $f_{r}: A \rightarrow B$ be an arbitrary constant function. Assume that $f_{i+1}: A^{n-n_{i+1}} \rightarrow B$ has been defined $(0 \leq i<r)$. Define $f_{i}: A^{n-n_{i}} \rightarrow B$ as follows. Let $g_{i}: A^{n-n_{i}} \rightarrow B$ be a function equivalent to $f_{i+1}$, i.e., $g_{i}$ is obtained from $f_{i+1}$ by adding $n_{i+1}-n_{i}$ inessential variables. Let $h_{i}: A^{n-n_{i}} \rightarrow B$ be an arbitrary nonconstant function such that $h_{i}\left(a_{1}, \ldots, a_{n-n_{i}}\right)=0$ whenever $a_{j}=a_{j^{\prime}}$ for some $j \neq j^{\prime}$. Set $f_{i}:=g_{i}+h_{i}$. It is easy to verify that $f_{i}$ depends on all of its variables. Moreover, the strict simple minors of $f_{i}$ are precisely the simple minors of $f_{i+1}$ (including $f_{i+1}$ itself). Therefore $f_{i+1}$ is the unique lower cover of $f_{i}$.

Setting $f:=f_{0}$, we have that the simple minors of $f$ are precisely the functions $f, f_{1}, \ldots, f_{r}$, and they comprise a covering sequence $f \succ f_{1} \succ f_{2} \succ \cdots \succ f_{r}$. Since ess $f_{\ell}=n-n_{\ell}$, we conclude that $\operatorname{gap}(f, \ell)=n_{\ell}$ for every $0 \leq \ell \leq r$, as desired.

\section{Concluding Remarks}

The parametrized arity gap constitutes a tool for tackling yet another natural problem pertaining to the effect of variable identification on the number of essential variables of a function. Given a function $f: A^{n} \rightarrow B$ and an integer $p \geq 1$, what is the smallest number $m$ such that any $m$ successive identifications of essential variables result in the loss of at least $p$ essential variables? Let us denote this smallest number by $\operatorname{pag}(f, p)$. As the reader may now realise, $\operatorname{pag}(f, p)$ is the smallest $\ell$ for which $\operatorname{gap}(f, \ell) \geq p$.

Example 4.1. Consider the 6-ary function $f$ of Example 3.2. We can read off of Figure 1 that

$$
\begin{array}{lll}
\operatorname{pag}(f, 1)=1, & \operatorname{pag}(f, 2)=2, & \operatorname{pag}(f, 3)=2, \\
\operatorname{pag}(f, 4)=3, & \operatorname{pag}(f, 5)=4, & \operatorname{pag}(f, 6)=4 .
\end{array}
$$

We may also consider similar problems when we perform several simultaneous identifications of variables. Following the formalism of Willard [16], we view functions of several variables as maps $f: A^{V} \rightarrow B$, where $V \subseteq\left\{x_{i}: i \in \mathbb{N}\right\}$. The cardinality of $V$ is called the arity of $f$. In this framework, a function $g: A^{W} \rightarrow B$ is a simple minor of $f: A^{V} \rightarrow B$, if there exists a map $\alpha: V \rightarrow W$ such that $g(\mathbf{a})=f(\mathbf{a} \circ \alpha)$ for all $\mathbf{a} \in A^{W}$.

We denote the set of all equivalence relations on a set $V$ by $\operatorname{Eq}(V)$. Given an equivalence relation $\theta \in \operatorname{Eq}(V)$, denote the canonical surjection by $v_{\theta}: V \rightarrow V / \theta$. For a function $f: A^{V} \rightarrow B$, we define the function $f^{\theta}: A^{V / \theta} \rightarrow B$ by the rule $f^{\theta}(\mathbf{a})=f\left(\mathbf{a} \circ v_{\theta}\right)$, and we say that $f^{\theta}$ is obtained from $f$ by block identification of variables through $\theta$. We informally identify $V / \theta$ with any one of its distinct representatives; in this way $f^{\theta}$ is a simple minor of $f$, and every simple minor of $f$ is equivalent to $f^{\psi}$ for some $\psi \in \operatorname{Eq}(V)$. The number of variables identified through $\theta$ is

$$
e(\theta):=\sum_{X \in V / \theta}(|X|-1)=|V|-|V / \theta| .
$$

Assuming that $f$ depends on all of its variables, i.e., ess $f=|V|$, we have that ess $f^{\theta} \leq$ $|V / \theta|=|V|-e(\theta)=\operatorname{ess} f-e(\theta)$.

Now we can define the analogue of the parametrized arity gap for block identification of variables. For a function $f: A^{V} \rightarrow B$ with ess $f=|V|=n$ and for an integer $\ell$ such that $0 \leq \ell \leq n-1$, we define

$$
\mathrm{b}-\operatorname{gap}(f, \ell):=\min _{\substack{\theta \in \operatorname{Eq}(V) \\ e(\theta)=\ell}}\left(\operatorname{ess} f-\operatorname{ess} f^{\theta}\right) .
$$

Note that $\mathrm{b}$-gap $(f, 0)=0$ and $\mathrm{b}-\operatorname{gap}(f, 1)=\operatorname{gap} f$ for every function $f$. It is also clear that $\ell \leq \operatorname{b-gap}(f, \ell) \leq n$ for every $0 \leq \ell \leq n-1$, and b-gap $(f, \ell) \leq \operatorname{gap}(f, \ell)$ for every $\ell$ for which $\operatorname{gap}(f, \ell)$ is defined. 
Let $H(f):=\{\operatorname{ess} f-\operatorname{ess} g: g \leq f\}$. It is clear that $\{\operatorname{b-gap}(f, \ell): 0 \leq \ell \leq n-1\} \subseteq$ $H(f)$.

Proposition 4.2. Let $f: A^{V} \rightarrow B$ be a function such that $\operatorname{ess} f=|V|=n$. Then b-gap $(f, \ell)=\min \{m \in H(f): m \geq \ell\}$, for all $0 \leq \ell \leq n-1$.

Proof. Let $\ell \in\{0, \ldots, n-1\}$. Assume first that $\ell \in H(f)$. Then there exists an equivalence relation $\theta \in \mathrm{Eq}(V)$ such that $\operatorname{ess} f^{\theta}=n-\ell$. It clearly holds that $e(\theta) \leq \ell$. We can construct an equivalence relation $\zeta \in \mathrm{Eq}(V)$ such that $e(\zeta)=\ell$ and $f^{\zeta} \equiv f^{\theta}$. Namely, we merge the blocks of $\theta$ that are indexing the inessential variables of $f^{\theta}$, if any, and we merge the resulting block with another block of $\theta$. We leave it for the reader to verify that $\zeta$ indeed satisfies $e(\zeta)=\ell$ and $f^{\zeta} \equiv f^{\theta}$. This construction shows that $\mathrm{b}-\operatorname{gap}(f, \ell) \leq \ell$. On the other hand, $\mathrm{b}-\operatorname{gap}(f, \ell) \geq \ell$, so we conclude that b-gap $(f, \ell)=\ell=\min \{m \in H(f): m \geq \ell\}$.

Assume then that $\ell \notin H(f)$. Since $\ell \leq \mathrm{b}-\operatorname{gap}(f, \ell) \in H(f)$, we have in fact that $\ell<\mathrm{b}$-gap $(f, \ell)$; moreover, b-gap $(f, \ell) \geq t$ for $t:=\min \{m \in H(f): m \geq \ell\}$. Since $\mathrm{b}-\mathrm{gap}(f, \cdot)$ is a monotone increasing function in its second argument, and b-gap $(f, t)=t$ by the first part of this proof, we have

$$
t \leq \mathrm{b}-\operatorname{gap}(f, \ell) \leq \mathrm{b}-\operatorname{gap}(f, t)=t,
$$

that is, $\operatorname{b-gap}(f, \ell)=t=\min \{m \in H(f): m \geq \ell\}$.

Example 4.3. Consider the 6-ary function $f$ of Example 3.2. We can read off of Figure 1 that $H(f)=\{0,1,2,3,4,6\}$ and

$$
\begin{aligned}
& \text { b-gap }(f, 1)=1, \quad \text { b-gap }(f, 2)=2, \quad \text { b-gap }(f, 3)=3 \text {, } \\
& \text { b-gap }(f, 4)=4, \quad \text { b-gap }(f, 5)=6 \text {. }
\end{aligned}
$$

We can still consider an analogue of the problem stated in the first paragraph of this section. Given a function $f: A^{V} \rightarrow B$ that depends on all of its variables and an integer $p \geq 1$, what is the smallest number $m$ such that block identification of variables of $f$ through every equivalence relation $\theta$ on $V$ with $e(\theta)=m$ results in the loss of at least $p$ essential variables? Let us denote this smallest number by b-pag $(f, p)$. It is again clear that b-pag $(f, p)$ is the smallest $\ell$ for which b-gap $(f, \ell) \geq p$. In other words, b-pag $(f, 0)=0$ and b-pag $(f, p)=\max \{m \in H(f): m<p\}+1$ for $1 \leq p \leq n$.

Example 4.4. Consider the 6-ary function $f$ of Example 3.2 We can determine from the values of b-gap $(f, \ell)$ listed in Example 4.3, or we can easily read off of Figure 1 that

$$
\begin{aligned}
& \text { b-pag }(f, 1)=1, \quad \text { b-pag }(f, 2)=2, \quad \text { b-pag }(f, 3)=3 \text {, } \\
& \text { b-pag }(f, 4)=4, \quad \text { b-pag }(f, 5)=5, \quad \text { b-pag }(f, 6)=5 \text {. }
\end{aligned}
$$

\section{REFERENCES}

[1] Berman, J., Kisielewicz, A.: On the number of operations in a clone. Proc. Amer. Math. Soc. 122, 359-369 (1994)

[2] Bouaziz, M., Couceiro, M., Pouzet, M.: Join-irreducible Boolean functions. Order 27, 261-282 (2010)

[3] Couceiro, M.: On the lattice of equational classes of Boolean functions and its closed intervals. J. Mult.-Valued Logic Soft Comput. 18, 81-104 (2008)

[4] Couceiro, M., Lehtonen, E.: On the effect of variable identification on the essential arity of functions on finite sets. Int. J. Found. Comput. Sci. 18, 975-986 (2007)

[5] Couceiro, M., Lehtonen, E.: Generalizations of Świerczkowski's lemma and the arity gap of finite functions. Discrete Math. 309, 5905-5912 (2009)

[6] Couceiro, M., Lehtonen, E., Waldhauser, T.: The arity gap of order-preserving functions and extensions of pseudo-Boolean functions. Discrete Appl. Math. 160, 383-390 (2012)

[7] Couceiro, M., Lehtonen, E., Waldhauser, T.: Decompositions of functions based on arity gap. Discrete Math. 312, 238-247 (2012)

[8] Couceiro, M., Lehtonen, E., Waldhauser, T.: On the arity gap of polynomial functions. arXiv:1104.0595 (2011)

[9] Couceiro, M., Pouzet, M.: On a quasi-ordering on Boolean functions. Theoret. Comput. Sci. 396, 71-87 (2008)

[10] Lehtonen, E.: Descending chains and antichains of the unary, linear, and monotone subfunction relations. Order 23, 129-142 (2006) 
[11] Lehtonen, E., Szendrei, Á.: Equivalence of operations with respect to discriminator clones. Discrete Math. 309, 673-685 (2009)

[12] Pippenger, N.: Galois theory for minors of finite functions. Discrete Math. 254, 405-419 (2002)

[13] Salomaa, A.: On essential variables of functions, especially in the algebra of logic. Ann. Acad. Sci. Fenn. Ser. A I. Math. 339, 3-11 (1963)

[14] Shtrakov, S., Koppitz, J.: On finite functions with non-trivial arity gap. Discuss. Math. Gen. Algebra Appl. 30, 217-245 (2010)

[15] Wang, C.: Boolean minors. Discrete Math. 141, 237-258 (1991)

[16] Willard, R.: Essential arities of term operations in finite algebras. Discrete Math. 149, 239-259 (1996)

[17] Zverovich, I.E.: Characterizations of closed classes of Boolean functions in terms of forbidden subfunctions and Post classes. Discrete Appl. Math. 149, 200-218 (2005)

(M. Couceiro) Mathematics Research Unit, University of Luxembourg, 6, Rue Richard Coudenhove-Kalergi, L-1359 Luxembourg, Luxembourg

E-mail address: miguel.couceiro@uni.lu

(E. Lehtonen) Computer Science and Communications Research Unit, University of Luxembourg, 6, Rue Richard Coudenhove-Kalergi, L-1359 Luxembourg, Luxembourg

E-mail address: erkko.lehtonen@uni.lu

(T. Waldhauser) University of Luxembourg, 6, Rue Richard Coudenhove-Kalergi, L-1359 Luxembourg, Luxembourg and Bolyai Institute, University of Szeged, Aradi vértanúk tere

1, H-6720 Szeged, Hungary

E-mail address: twaldha@math.u-szeged.hu 